\title{
SIMULAÇÃO DO BALANÇO DE ÁGUA NO SOLO CULTIVADO COM TRIGO COM MODIFICAÇÃO DE DOIS MODELOS ${ }^{(1)}$
}

\author{
Cleber Maus Alberto ${ }^{(2)}$, Nereu Augusto Streck ${ }^{(3)}$, Felipe Brendler \\ Oliveira $^{(4)}$, Alencar Junior Zanon ${ }^{(4)} \&$ Diego Garrido Pinheiro ${ }^{(4)}$
}

\begin{abstract}
RESUMO
O trigo é a principal cultura de inverno do estado do Rio Grande do Sul e o cálculo do balanço de água no solo é parte importante de modelos de crescimento, desenvolvimento e rendimento de culturas. O objetivo deste trabalho foi obter melhor estimativa do balanço de água no solo cultivado com trigo, modificando dois modelos de balanço de água nesse solo. Mediu-se o conteúdo de água no solo pelo método gravimétrico durante a estação de cultivo de dois cultivares de trigo em três datas de semeadura, em Santa Maria - RS, e a água disponível para a cultura foi representada pela fração de água no solo disponível para as plantas (FADS). O desempenho das versões originais e modificadas dos modelos de balanço de água no solo de Campbell \& Diaz e de Amir \& Sinclair foi avaliado pela raiz do quadrado médio do erro (RQME). O modelo de Campbell \& Diaz modificado é mais realístico e com maior possibilidade de desempenho satisfatório em regióes de clima distinto daquele em que foi desenvolvido, mas o modelo de Amir \& Sinclair modificado estimou melhor a água disponível no solo para a cultura do trigo na região do estudo. A profundidade máxima do sistema radicular de 0,30 m é mais apropriada para a simulação da fração de água disponível no solo, para a unidade de mapeamento de solo São Pedro.
\end{abstract}

Termos de indexação: Triticum aestivum L., fenologia, ambiente, estresse hídrico.

\footnotetext{
(1) Parte da Tese de Doutorado do primeiro autor apresentada ao Programa de Pós-Graduação em Engenharia Agrícola, Universidade Federal de Santa Maria - UFSM. Financiado com recursos da CAPES. Recebido para publicação em agosto de 2008 e aprovado em junho de 2009.

(2) Professor Substituto do Departamento de Fitotecnia, Universidade Federal de Santa Maria - UFSM. Camobi, CEP 97105-900 Santa Maria (RS). E-mail: cleberalb@yahoo.com.br

(3) Professor Adjunto do Departamento de Fitotecnia, UFSM. E-mail: nstreck2@yahoo.com.br

(4) Acadêmico do Curso de Agronomia, UFSM.
} 


\title{
SUMMARY: SIMULATING WATER BALANCE IN SOIL UNDER WHEAT BY TWO MODIFIED MODELS
}

\begin{abstract}
Wheat is the main winter crop in Rio Grande do Sul State, Brazil, and the calculation of soil water balance is an important element in simulation models for crop growth, development and yield. The objective of this study was to improve the water balance estimation in soil under wheat by two modified models. The soil water content was measured by the gravimetric method during the growing season of two wheat cultivars sown on three dates in Santa Maria, RS, Brazil. Soil water for crop was represented as the fraction of plant-available soil water (FASW). The performance of the original and the modified version of two water balance models, namely the Campbell \& Diaz model and the Amir \& Sinclair model, was evaluated by the root of the mean square error (RMSE). The modified Campbell \& Diaz model is more realistic and has a greater potential for performing well in regions other than where it was developed, while the modified Amir \& Sinclair model simulated the available soil water for a wheat crop better in the study region. A maximum root depth of $0.3 \mathrm{~m}$ is most appropriate to simulate the fraction of plant-available water in the São Pedro soil unit.
\end{abstract}

Index terms: Triticum aestivum L., phenology, environment, water stress.

\section{INTRODUÇÃO}

A água é o principal fator abiótico que causa variação no rendimento das culturas na maior parte das áreas agrícolas (Eitzinger et al., 2003; Okuyama et al., 2004; Streck, 2004). Assim, a estimativa da água disponível no solo para as plantas é parte importante de modelos de crescimento, desenvolvimento e rendimento das culturas agrícolas (Dardanelli et al., 2004). No Rio Grande do Sul, o trigo é a principal cultura de inverno.

Há na literatura um grande número de modelos que descrevem o balanço de água no solo das culturas agrícolas com vários graus de complexidade. O modelo de Campbell \& Diaz (1988) é um desses modelos, desenvolvido em Davenport, Washington, USA, uma região de clima DSb pela classificação de Köppen (clima continental com verões quentes e chuvas no inverno). É intermediário em termos de complexidade, necessita de um reduzido número de dados de entradae é composto de relações matemáticas simplificadas entre a cultura e o solo. Nesse modelo, o solo é dividido em camadas e o crescimento radicular da planta de trigo é simulado utilizando a data de maturidade fisiológica da cultura, que é um dado de entrada. Portanto, este modelo só pode ser utilizado quando o ciclo de desenvolvimento da cultura estiver completo ou for conhecido com antecedência, o que constitui uma desvantagem para seu uso prático durante o ciclo de desenvolvimento da cultura.

Outro modelo de simulação de balanço de água no solo cultivado com trigo é o modelo de Amir \& Sinclair (1991b), desenvolvido em Gilat, Israel, uma região de clima BSh pela classificação de Köppen (clima semiárido quente com chuvas no inverno). Uma vantagem desse modelo é que necessita de poucos atributos físicos do solo para o seu funcionamento (conteúdo volumétrico de água no solo a tensões de 0,1 e $-1,5 \mathrm{MPa}$ ), mas a desvantagem é que a profundidade do sistema radicular é assumida constante do início (semeadura) ao fim da simulação (maturidade fisiológica), o que não corresponde à realidade do ponto de vista biológico, pois nas fases iniciais de desenvolvimento da cultura apenas uma pequena faixa de solo é explorada pelo sistema radicular da planta. Nesse modelo ainda, o desenvolvimento do trigo é simulado usando a soma térmica (graus-dia), que nem sempre é o método que melhor estima as datas de ocorrência dos estádios de desenvolvimento do trigo (Streck et al., 2003a,b).

Uma possibilidade para contornar a necessidade do conhecimento prévio da data de maturidade fisiológica no modelo de Campbell \& Diaz (1988) é vincular o modelo de crescimento radicular a um modelo de desenvolvimento da cultura. Há vários modelos de simulação do desenvolvimento em trigo (Streck et al., 2003a,b), destacando-se o de Wang \& Engel - WE (Wang \& Engel, 1998), que é composto por funções de resposta não lineares combinadas de forma multiplicativa, uma vantagem em relação aos modelos que usam soma térmica. Já no modelo de Amir \& Sinclair (1991a,b), a inclusão de um modelo de desenvolvimento não linear e de um modelo de simulação do crescimento radicular no perfil do solo pode melhorar a estimativa do balanço de água do solo. A hipótese neste estudo é que tais alterações tornam esses dois modelos de simulação do balanço de água do solo cultivado com trigo mais realistas do que as versões originais, melhorando, assim, sua capacidade preditiva em regiões distintas daquelas em que os modelos foram calibrados. 
Os objetivos deste trabalho foram: (a) obter melhor estimativa do balanço de água no solo, introduzindo o modelo de desenvolvimento de Wang \& Engel (1998) em substituição à data de maturidade fisiológica do trigo no modelo de Campbell \& Diaz (1988); e (b) introduzir um modelo de crescimento radicular e substituir o modelo de desenvolvimento da soma térmica pelo modelo WE no modelo de Amir \& Sinclair (1991a,b).

\section{MATERIAL E MÉTODOS}

Um experimento de campo foi realizado em Santa Maria (29 $43^{\prime} \mathrm{S}, 53^{\circ} 43^{\prime} \mathrm{W}$ e altitude de $95 \mathrm{~m}$ ), RS. O local pertence à unidade de mapeamento São Pedro (Argissolo Vermelho Distrófico Arênico). Santa Maria está localizada na região fisiográfica da Depressão Central do Rio Grande do Sul, e o clima, segundo a classificação de Köppen, é Cfa, subtropical úmido, sem estação seca definida com verões quentes (Moreno, 1961).

O preparo do solo foi realizado de maneira convencional com gradagens. Na adubação de base foram aplicados $300 \mathrm{~kg}^{\text {ha-1 }}$ da formulação 5-20-20 no sulco de semeadura de acordo com a análise química do solo, seguindo as recomendações de adubação e calagem para os Estados do Rio Grande do Sul e Santa Catarina (SBCS/CQFS, 2004). Foi realizada adubação nitrogenada em cobertura, na quantidade de $80 \mathrm{~kg} \mathrm{ha}^{-1} \mathrm{de} \mathrm{N}$, parcelada em duas épocas, a primeira no início do perfilhamento e a segunda no estádio de espigueta terminal.

Utilizaram-se os cultivares de trigo BRS 177 (EMBRAPA) e CEP 52 (FUNDACEP), recomendados para o Rio Grande do Sul. Foram realizadas duas semeaduras do cultivar BRS 177 (4/09/2006 e 18/04/ 2007) e uma do CEP 52 (7/7/2006). Foram determinadas as datas de ocorrência dos estádios de emergência, antese e maturidade fisiológica de acordo com os critérios descritos em Zadoks et al. (1974). Cada parcela foi constituída de quatro linhas espaçadas a $0,20 \mathrm{~m}$ com $2,40 \mathrm{~m}$ de comprimento e área de $1,92 \mathrm{~m}^{2}$. A densidade de plantas foi 330 plantas $\mathrm{m}^{-2}$, que é uma densidade de plantas comumente encontrada em lavouras comerciais de trigo no Rio Grande do Sul.

O conteúdo de água no solo foi medido pelo método gravimétrico nas camadas de $0-0,10,0,10-0,30$ e de $0,30-0,60 \mathrm{~m}$. Foram coletadas quatro amostras de solo para cada camada na linha central de cada parcela, em um intervalo de aproximadamente 14 dias, no período entre a emergência e a antese. Evitou-se coletar amostras de solos durante o período de chuva e nos dias imediatamente subsequentes, quando o conteúdo de água estava próximo e acima da capacidade de campo, situação que não é de interesse neste estudo. Como a cultura do trigo em regiões subtropicais semelhante ao local deste estudo, tem boa parte do seu ciclo de desenvolvimento durante o inverno quando a demanda evaporativa do ar é baixa e devido ao regime pluviométrico isoígro (chuvas distribuídas em todas as estações do ano) neste local, ocorrem, durante o inverno e a primavera, chuvas semanais que podem durar de um a vários dias. Leva vários dias após a chuva para o conteúdo de água do solo se afastar da capacidade de campo. Também, no período de 86 a 132 e 132 a 165 dias após a emergência (DAE), na semeadura de 18/04/2007 do cultivar BRS 177, ocorreram problemas na amostragem de solo, e optouse por não utilizar esses dados, o que acarretou períodos de maior distância entre as datas de amostragem de solo para esta data de semeadura e cultivar, mas que não comprometeram o objetivo do trabalho. As amostras de solo coletadas foram levadas à estufa a $105 \pm 5{ }^{\circ} \mathrm{C}$, onde permaneceram até peso constante. Determinou-se a densidade do solo pelo método do anel volumétrico, coletando-se quatro amostras nas camadas de $0-0,10,0,10-0,30$ e $0,30-0,60 \mathrm{~m}$. Com esses dados, foi possível a obtenção da umidade volumétrica do solo nas diferentes camadas (Embrapa, 1997).

Os valores de conteúdo volumétrico de água na capacidade de campo $\left(\mathrm{CC}, \mathrm{m}^{3} \mathrm{~m}^{-3}\right)$ e no ponto de murcha permanente (PMP, $\mathrm{m}^{3} \mathrm{~m}^{-3}$ ) foram estimados pela equação de pedotransferência proposta por Urach (2007), a partir de teores médios de argila para o solo da unidade de mapeamento São Pedro descritos em Galvagni (1998).

A profundidade máxima do sistema radicular (RDmax) foi medida abrindo-se uma trincheira em uma parcela do cultivar BRS 177 no dia 30/08/2007 (uma semana após a antese deste cultivar, semeado no dia 18/04/2007). Da semeadura até o dia 30/08/2007, a precipitação acumulada foi de $461,1 \mathrm{~mm}$, indicando boa disponibilidade hídrica durante este período. Assim, teve-se segurança de que o crescimento radicular não teve limitação por água nem por nutrientes.

Os elementos meteorológicos (temperatura máxima e mínima, brilho solar real e precipitação pluvial) durante o período experimental foram coletados na Estação Climatológica Principal do $8^{\circ}$ Distrito de Meteorologia (DISME) - Ministério da Agricultura, localizada a aproximadamente $100 \mathrm{~m}$ do local de cultivo.

Os dados meteorológicos necessários e usados para rodar o modelo de Campbell \& Diaz (1988) foram temperatura mínima $\left(\mathrm{Tn},{ }^{\circ} \mathrm{C}\right)$ e máxima $\left(\mathrm{Tx},{ }^{\circ} \mathrm{C}\right)$ diária do ar e a precipitação diária $\left(\mathrm{m} \mathrm{dia}^{-1}\right)$, e os dados de solo foram conteúdo de água do solo na capacidade de campo (CC), conteúdo de água do solo no ponto de murcha permanente (PMP) e conteúdo de água do solo seco ao ar livre (ADSS, um terço do PMP). Os dados da cultura necessários no modelo original são a data de semeadura, data de emergência, data de maturidade fisiológica e a RDmax (m).

O crescimento de raízes no modelo original de Campbell \& Diaz (1988) foi calculado como:

$\mathrm{RD}=[\mathrm{PES}+($ RDmax-PES $)] \times\left[\frac{1}{1+44,2 \times \exp (-8,5 \mathrm{x}(\mathrm{DOY}-\mathrm{DEM}) \div(\text { DMF-DEM })}\right)$ 
em que $\mathrm{RD}$ é a profundidade do sistema radicular (m) em determinado dia do ano (DOY), RDmax é a profundidade máxima $(\mathrm{m})$ que o sistema radicular pode alcançar, PES é a profundidade em que ocorreu a evaporação (neste estudo 0,1 m; Silva et al., 2006), DEM é o dia do ano em que ocorre a emergência da cultura, e DMF é o dia do ano em que ocorre a maturidade fisiológica da cultura.

O modelo de Campbell \& Diaz (1988) calcula o conteúdo atual de água no solo em cada camada diariamente. Do ponto de vista biológico, no entanto, é mais interessante representar a quantidade de água no solo que está disponível para a planta. Neste trabalho foi considerada a fração de água disponível no solo para a planta (Streck \& Alberto, 2006). O índice FADS varia de zero a 1 e foi calculado como:

$$
\mathrm{FADS}=\frac{\mathrm{CAA}-\mathrm{PMP}}{\mathrm{CC}-\mathrm{PMP}}
$$

em que CAA é o conteúdo atual de água no solo $\left(\mathrm{m}^{3} \mathrm{~m}^{-3}\right)$, CC é o conteúdo de água no solo na capacidade de campo $\left(\mathrm{m}^{3} \mathrm{~m}^{-3}\right)$ e PMP é o conteúdo de água no solo no ponto de murcha permanente $\left(\mathrm{m}^{3} \mathrm{~m}^{-3}\right)$.

Como o modelo de Campbell \& Diaz (1988) necessita do conhecimento prévio da data de ocorrência da maturidade fisiológica para o cálculo da RD (Equação 1), propos-se, neste estudo, a substituição da DEM e DMF pelo modelo de desenvolvimento WE para trigo (Wang \& Engel, 1998) modificado por Streck et al. (2003b). Com esta modificação, o RD é calculado por:

$$
\mathrm{RD}=[\mathrm{PES}+(\mathrm{RDmax}-\mathrm{PES})] \times\left[\frac{1}{1+44,2 \times \exp \left(-8,5 \times\left(\frac{\mathrm{D}}{2}\right)\right)}\right]
$$

em que $\mathrm{D}$ é o índice de desenvolvimento da cultura do trigo, calculado pelo somatório da taxa diária de desenvolvimento calculado pelo modelo WE modificado por Streck et al. (2003b), com coeficientes ajustados para os cultivares BRS 177 e CEP 52 (Alberto, 2008). As funções de vernalização [f(v)] e temperatura [f(T)] no modelo WE foram calculadas usando-se dados diários de temperatura máxima (Tx) e mínima (Tn). Depois disso, calculou-se a média aritmética dessas funções.

Os dados meteorológicos necessários para rodar o modelo de Amir \& Sinclair (1991a,b) foram temperaturas mínima $\left(\mathrm{Tn},{ }^{\circ} \mathrm{C}\right)$ e máxima $\left(\mathrm{Tx},{ }^{\circ} \mathrm{C}\right)$ diária do ar e precipitação pluvial diária $\left(\mathrm{mm} \mathrm{dia}^{-1}\right)$. Os dados de solo foram conteúdo de água do solo na capacidade de campo (CC) e no ponto de murcha permanente (PMP). Os dados da cultura usados no modelo original foram número final de folhas (NFF), filocrono $\left({ }^{\circ} \mathrm{C} \mathrm{dia}^{-1 /} / \mathrm{folha}\right.$ ), o ciclo de desenvolvimento da cultura (médio ou precoce), a profundidade do solo em que ocorreu a evaporação (PS1) e a profundidade de solo explorada pelo sistema radicular (PS2).
A capacidade total de água que pode ser armazenada no solo e que fica disponível para a planta depende da profundidade do sistema radicular. No modelo de Amir \& Sinclair (1991b), o solo é dividido em duas camadas. Na PS1, ocorre a evaporação de água do solo e a transpiração de água pela planta. $\mathrm{Na}$ PS2, ocorre apenas a transpiração de água pela planta. A FADS foi calculada pela equação (2).

No modelo original de Amir \& Sinclair (1991b), a PS2 é considerada constante do início ao final do ciclo de desenvolvimento da cultura. Para simular o crescimento radicular de forma mais realista, proposse, neste estudo, a substituição da PS2 pelo RD calculado com a equação (3). O NFF utilizado para a versão original e modificada de Amir \& Sinclair (1991a,b) foi de 10 folhas para o cultivar CEP 52 na semeadura de 7/07/2006, 10 folhas para o cultivar BRS 177 na semeadura de 4/09/2006 e de 11 folhas para o cultivar BRS 177 na semeadura de 18/04/2007. As simulações com todos os modelos foram iniciadas no dia 25/06/2006 ( $\mathrm{P}=30,7 \mathrm{~mm}), 11 / 08 / 2006$ $(\mathrm{P}=32 \mathrm{~mm})$ e $9 / 04 / 2007(\mathrm{P}=47,3 \mathrm{~mm})$ para as datas de semeadura de 7/07/2006, 4/09/2006 e 18/04/2007, respectivamente. Considerou-se que, nessas datas, $o$ perfil de solo estava na capacidade de campo, pressupondo-se que, após uma precipitação pluvial acima de $30 \mathrm{~mm}$, o solo encontrava-se na capacidade de campo, em função de observações morfológicas no campo.

Para avaliar o desempenho das versões originais e modificadas dos dois modelos, foi utilizada a raiz do quadrado médio do erro (RQME), calculada como (Janssen \& Heuberger, 1995):

$$
\mathrm{RQME}=\sqrt{\left[\frac{1}{\mathrm{~N}} \sum_{\mathrm{i}=1}^{\mathrm{N}}\left(\mathrm{S}_{\mathrm{i}}-\mathrm{o}_{\mathrm{i}}\right)^{2}\right]}
$$

em que s é o valor simulado da FADS, o é o valor observado da FADS e N é o número de observações. Quanto menor o RQME, melhor é a simulação. Para análise de correlação entre os dados simulados e observados da FADS, foi utilizado o teste não paramétrico de Spearman-R (Streck \& Alberto, 2006).

Nos modelos com simulação do crescimento radicular, a FADS é dependente da profundidade do sistema radicular em um dado momento. Portanto, para possibilitar a comparação entre dados observados e simulados, foi efetuada a média dos dados observados de conteúdo de água no solo em cada camada, sendo este valor dependente da profundidade do sistema radicular (RD) simulada no dia da coleta das amostras de solo.

\section{RESULTADOS}

A densidade do solo foi de $1.520,1.560$ e $1.400 \mathrm{~kg} \mathrm{~m}^{-3}$ nas camadas de $0-0,10,0,10-0,30$ e $0,30-0,60 \mathrm{~m}$ de 
profundidade, respectivamente. Os valores calculados pela equação de pedotransferência (Urach, 2007) de CC foram de $0,261 \mathrm{~m}^{3} \mathrm{~m}^{-3}$ (camadas de $0-0,10$ e 0,10 $0,30 \mathrm{~m}$ ) e $0,336 \mathrm{~m}^{3} \mathrm{~m}^{-3}$ (camada de $0,30-0,60 \mathrm{~m}$ ) e de PMP foram de $0,138 \mathrm{~m}^{3} \mathrm{~m}^{-3}$ (camadas de $0-0,10 \mathrm{e}$ $0,10-0,30 \mathrm{~m}$ ) e $0,184 \mathrm{~m}^{3} \mathrm{~m}^{-3}$ (camada de $0,30-0,60 \mathrm{~m}$ ).

Constatou-se a presença de raízes até a profundidade de $0,60 \mathrm{~m}$, porém as raízes eram raras a partir de $0,30 \mathrm{~m}$ de profundidade e concentradas em galerias decorrentes da macrofauna do solo. A baixa frequência de raízes em profundidades maiores do que $0,30 \mathrm{~m}$ pode ter sido favorecida pela maior densidade do solo na camada $0,10-0,30 \mathrm{~m}$ decorrente das sucessivas gradagens realizadas em cultivos anteriores nesta área. Quando se utiliza preparo de solo convencional com várias gradagens, pode ocorrer a compactação do solo, principalmente na camada de solo de $0,10-0,30 \mathrm{~m}$ de profundidade (Stone \& Silveira, 1999).

Os valores da FADS observados e simulados pelo modelo de Campbell \& Diaz (1988), versão original e modificada, para as profundidades máximas do sistema radicular (RDmax) de 0,30 e 0,60 m durante a estação de crescimento dos dois cultivares de trigo estão apresentados nas figuras 1,2 e 3 . O menor valor de RQME $(0,135)$ para o modelo de Campbell \& Diaz (1988) na data de semeadura de 7/07/2006 foi obtido utilizando o modelo de crescimento radicular original e RDmax de 0,30 m (Figura 1a) e o maior valor de
$\operatorname{RQME}(0,246)$ foi obtido utilizando o modelo modificado e RDmax de 0,60 m (Figura 1d).

Para a semeadura de 4/09/2006, o menor valor de RQME $(0,330)$ pelo modelo de Campbell \& Diaz (1988) foi obtido utilizando-se o modelo modificado com RDmax de $0,30 \mathrm{~m}$ (Figura $2 \mathrm{~b}$ ), e o maior valor de RQME $(0,367)$ foi com o modelo original com RDmax de $0,60 \mathrm{~m}$ (Figura 2c). Para a data de semeadura 18/04/2007, o menor valor de RQME $(0,358)$ foi simulado pelo modelo de Campbell \& Diaz (1988) modificado com RDmax de $0,30 \mathrm{~m}$ (Figura 3b), e o maior valor de RQME $(0,396)$ foi simulado com o modelo Campbell \& Diaz (1988) original com RDmax de 0,60 m (Figura 3c).

O teste não paramétrico de correlação Spearman R para o modelo de Campbell \& Diaz (1988) mostrou a menor correlação entre dados simulados e observados $\left(\mathrm{r}_{\mathrm{s}}=0,26, \mathrm{NS} ; \mathrm{n}=6\right)$, para a data de semeadura de 7/07/2006, com RDmax de 0,30 m tanto com o modelo original quanto com o modificado. A maior correlação $\left(r_{s}=0,43 ; \mathrm{NS} ; \mathrm{n}=6\right)$ foi obtida com o modelo modificado utilizando RDmax de 0,60 m (Figura 1).

As simulações com o modelo de Campbell \& Diaz (1988) para a semeadura de 4/09/2006 não apresentaram boa correlação para nenhum dos modelos testados (Figura 2). Para a semeadura de 18/04/2007 (Figura 3), a maior correlação foi obtida com o modelo modificado e RDmax de 0,60 m (0,94;

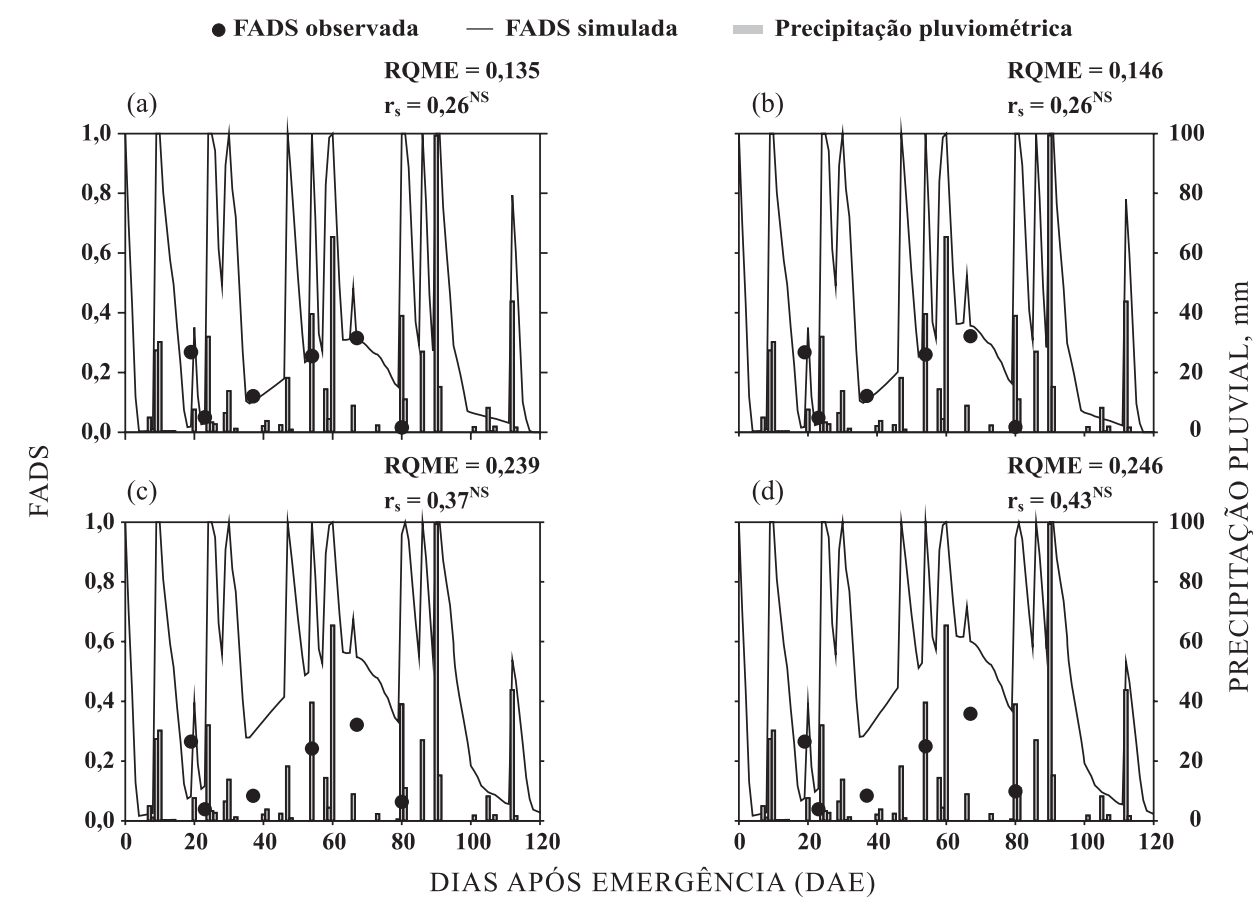

Figura 1. Fração de água disponível no solo (FADS) observada e simulada no perfil de solo onde ocorre crescimento radicular pelo modelo de Campbell \& Diaz (1988), com RDmax de 0,30 m, modelo original (a) e modificado (b), e para RDmax de $0,60 \mathrm{~m}$, modelo original (c) e modificado (d), e precipitação pluvial (P, mm), para a data de semeadura de 7/07/2006 do cultivar CEP 52. Santa Maria, RS. RQME: raiz do quadrado médio do erro, $r_{\mathrm{s}}$ : teste de correlação não paramétrico de Spearman R. NS: não significativo, *significativo a $5 \%$. 


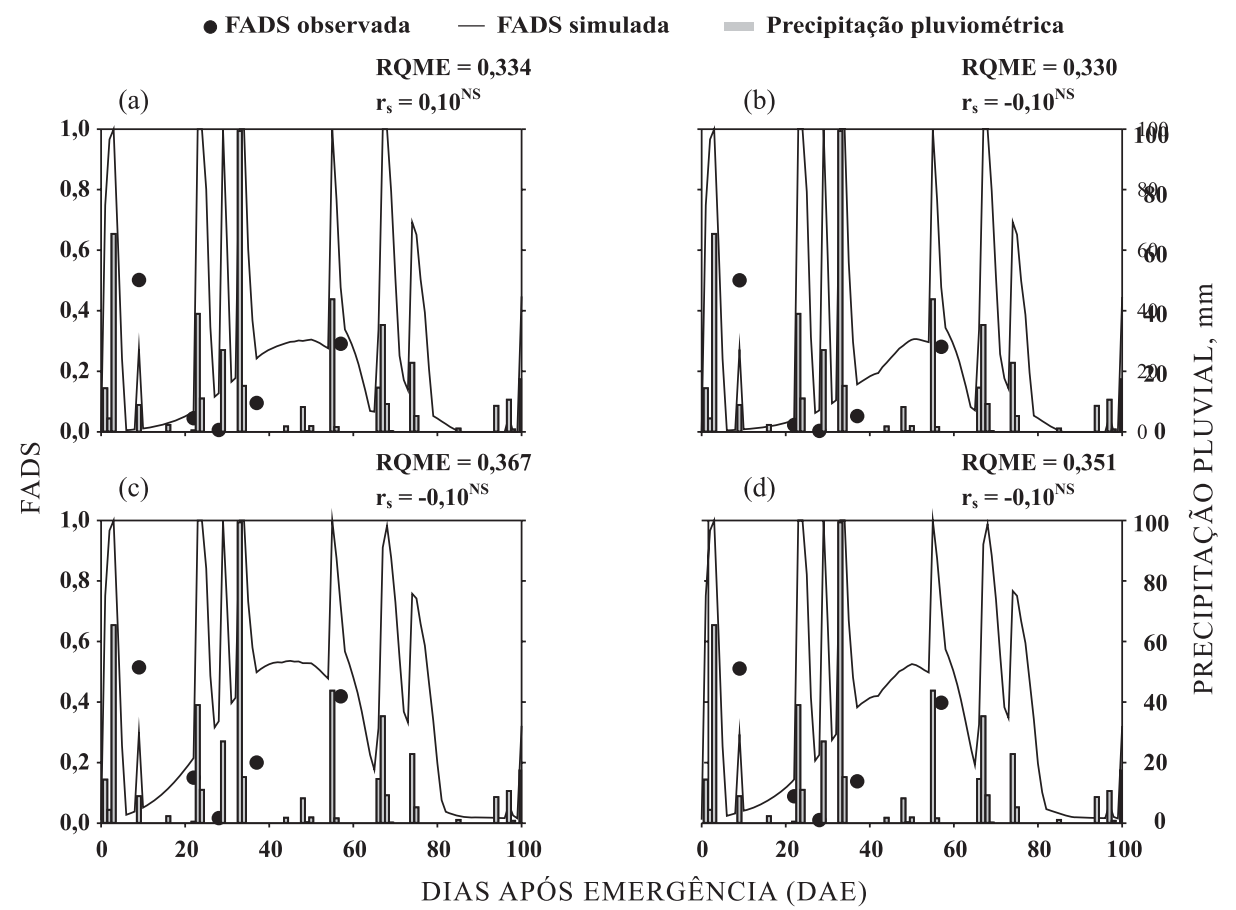

Figura 2. Fração de água disponível no solo (FADS) observada e simulada no perfil de solo onde ocorre crescimento radicular pelo modelo de Campbell \& Diaz (1988), com RDmax de 0,30 m, modelo original (a) e modificado (b), e para RDmax de $0,60 \mathrm{~m}$, modelo original (c) e modificado (d), e precipitação pluvial (P, mm), para a data de semeadura de 4/09/2006 do cultivar BRS 177. Santa Maria, RS. RQME: raiz do quadrado médio do erro, $r_{s}$ : teste de correlação não paramétrico de Spearman R. NS: não significativo, *significativo a $5 \%$.

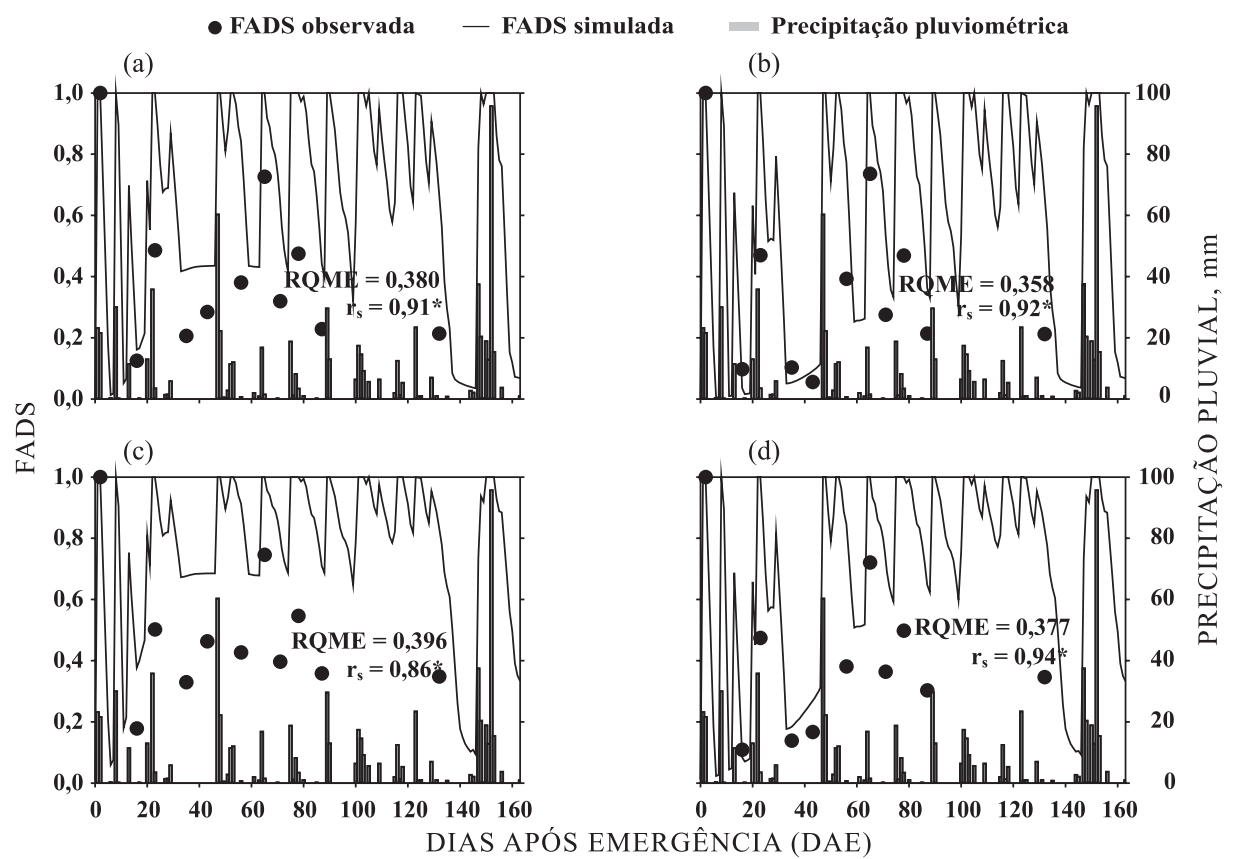

Figura 3. Fração de água disponível no solo (FADS) observada e simulada no perfil de solo onde ocorre crescimento radicular pelo modelo de Campbell \& Diaz (1988), com RDmax de 0,30 m, modelo original (a) e modificado (b), e para RDmax de $0,60 \mathrm{~m}$, modelo original (c) e modificado (d), e precipitação pluvial (P, mm), para a data de semeadura de 18/04/2007 do cultivar BRS 177. Santa Maria, RS. RQME: raiz do quadrado médio do erro, $r_{\mathrm{s}}$ : teste de correlação não paramétrico de Spearman R. NS: não significativo, *significativo a $5 \%$. 
$\mathrm{p}<0,05 ; \mathrm{n}=11$ ), e a menor correlação com RDmax de $0,60 \mathrm{~m}$ com o modelo de crescimento radicular original $\left(\mathrm{r}_{\mathrm{s}}=0,86 ; \mathrm{p}<0,05 ; \mathrm{n}=11\right)$.

Na simulação com o modelo de Amir \& Sinclair (1991a,b), na semeadura de 7/07/2006, o menor valor de $\operatorname{RQME}(0,111)$ foi obtido para a profundidade de 0,30 m utilizando o modelo modificado (Figura 4b), e o maior valor de RQME $(0,370)$ foi obtido para a profundidade de $0,60 \mathrm{~m}$, com o modelo original (Figura 4c). Na data de semeadura de 4/09/2006, o menor valor de RQME $(0,316)$ foi com o modelo original e profundidade de $0,60 \mathrm{~m}$ (Figura 5c), e o maior valor de $\operatorname{RQME}(0,412)$ foi com o modelo original e profundidade de 0,30 m (Figura 5a). Para a data de semeadura de 18/04/2007, o menor valor de RQME (0,234) foi com o modelo modificado de Amir \& Sinclair (1991a,b) e profundidade de 0,60 m (Figura 6d), e o maior valor de RQME $(0,326)$ foi com o modelo original e profundidade de 0,30 m (Figura 6a).

$\mathrm{Na}$ data de semeadura de 7/07/2006, a maior correlação entre a FADS simulada pelo modelo de Amir \& Sinclair (1991a,b) e observada $\left(\mathrm{r}_{\mathrm{s}}=1,00\right.$; $\mathrm{p}<0,05, \mathrm{n}=6$ ), foi obtida com o modelo modificado e RDmax de 0,30 m, e a menor correlação $\left(r_{\mathrm{s}}=0,60\right.$; $\mathrm{p}<0,05, \mathrm{n}=6$ ) foi obtida com o modelo original utilizando RDmax de 0,60 m (Figura 4). De maneira similar ao modelo de Campbell \& Diaz (1988), para a data de semeadura de 4/09/2006, a simulação da FADS pelo modelo de Amir \& Sinclair (1991a,b) não apresentou boa correlação para nenhum dos modelos testados (Figura 5), mas com os modelos de Amir \& Sinclair (1991a,b) modificados, com RDmax de 0,30 e $0,60 \mathrm{~m}$, as correlações pelo teste não paramétrico de Spearman $\mathrm{R}$ foram superiores $\left(\mathrm{r}_{\mathrm{s}}=0,35\right.$ e $\mathrm{r}_{\mathrm{s}}=0,34$, respectivamente) aos modelos originais $\left(r_{\mathrm{S}}=-0,10\right)$. $\mathrm{Na}$ semeadura de 18/04/2007, a maior correlação $\left(\mathrm{r}_{\mathrm{s}}=\right.$ 0,88; $\mathrm{p}<0,05 ; \mathrm{n}=11$ ) pelo teste de Spearman $\mathrm{R}$ foi com o modelo modificado de Amir \& Sinclair (1991a,b) e RDmax de 0,60 m, e a menor correlação $\left(r_{\mathrm{s}}=0,72\right.$; $\mathrm{p}<0,05 ; \mathrm{n}=11$ ) foi com o modelo original e RDmax de $0,60 \mathrm{~m}$ (Figura 6).

\section{DISCUSSÃO}

Os valores simulados de FADS com o modelo de Campbell \& Diaz (1988) foram sempre superiores aos observados, principalmente quando se considerou uma RDmax de 0,60 m. A distância entre os valores simulados com este modelo e os observados diminuiu quando se usou RDmax de 0,30 m, tanto para o modelo original quanto para o modelo modificado, principalmente na data de semeadura de 7/07/2006 (Figura 1), indicando que a concentração de raízes até a profundidade de $0,30 \mathrm{~m}$ observada no campo deve ser usada neste modelo.

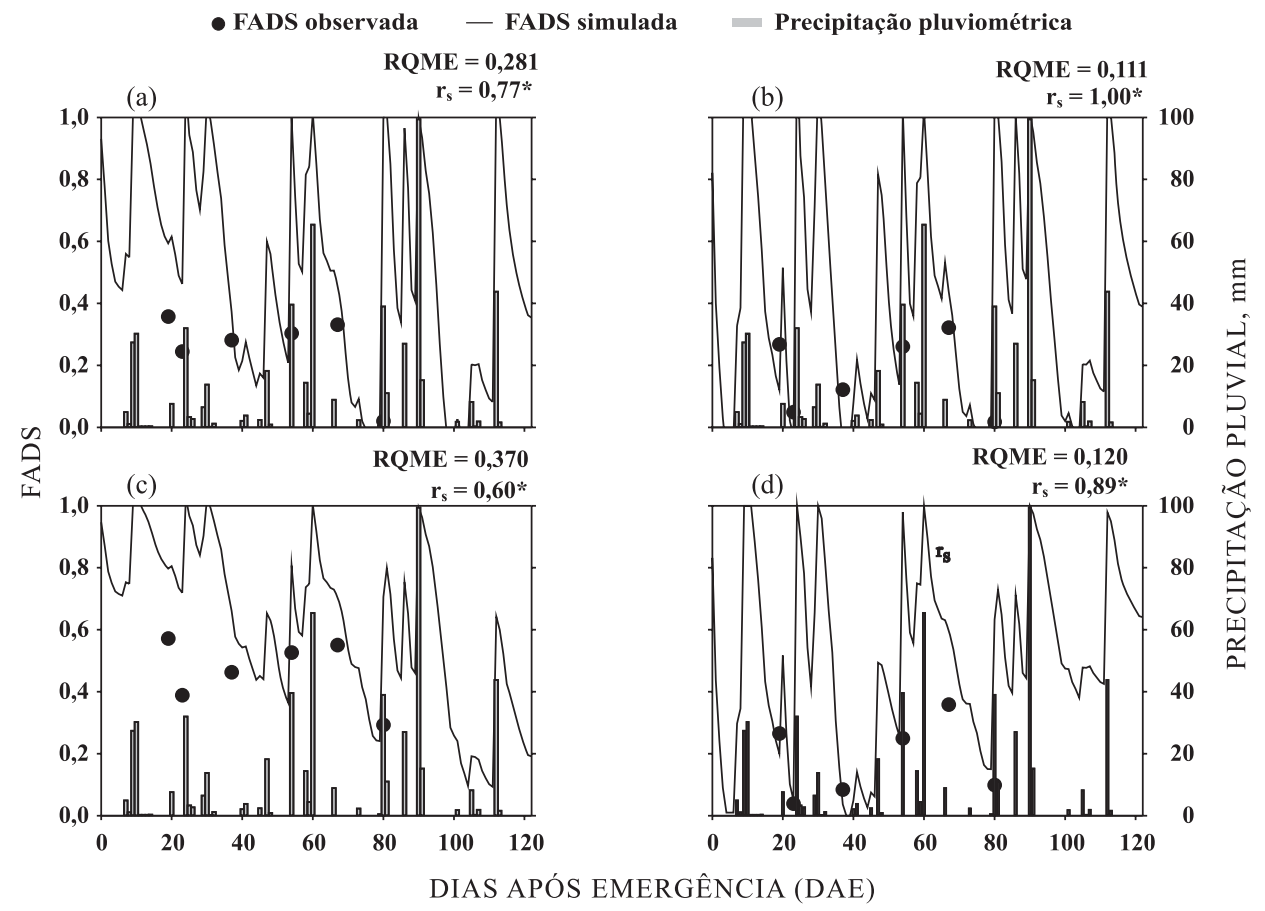

Figura 4. Fração de água disponível no solo (FADS) observada e simulada no perfil de solo onde ocorre crescimento radicular pelo modelo de Amir \& Sinclair (1991a,b) com RDmax de 0,30 m, modelo original (a) e modificado (b), e com RDmax de 0,60 m, modelo original (c) e modificado (d), e precipitação pluvial (P, mm), para a semeadura de 7/07/2006 do cultivar CEP 52. Santa Maria, RS. RQME: raiz do quadrado médio do erro, $r_{\mathrm{s}}$ : teste de correlação não paramétrico de Spearman R. NS: não significativo, *significativo a $5 \%$. 


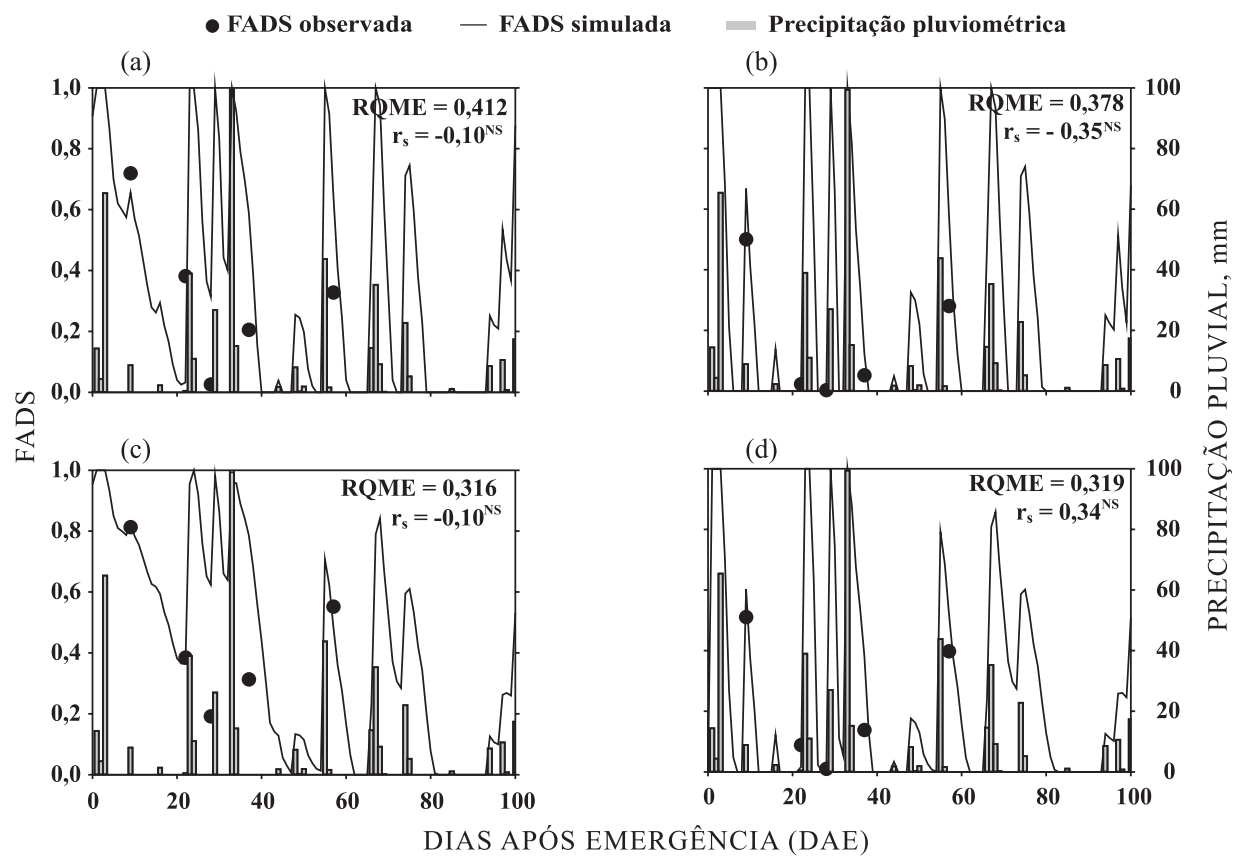

Figura 5. Fração de água disponível no solo (FADS) observada e simulada no perfil de solo onde ocorre crescimento radicular pelo modelo de Amir \& Sinclair (1991a,b) com RDmax de 0,30 m, modelo original (a) e modificado (b), e com RDmax de $0,60 \mathrm{~m}$, modelo original (c) e modificado (d), e precipitação pluvial (P, mm), para a semeadura de 4/09/2006 do cultivar BRS 177. Santa Maria, RS. RQME: raiz do quadrado médio do erro, $r_{\mathrm{s}}$ : teste de correlação não paramétrico de Spearman R. NS: não significativo, *significativo a $5 \%$.

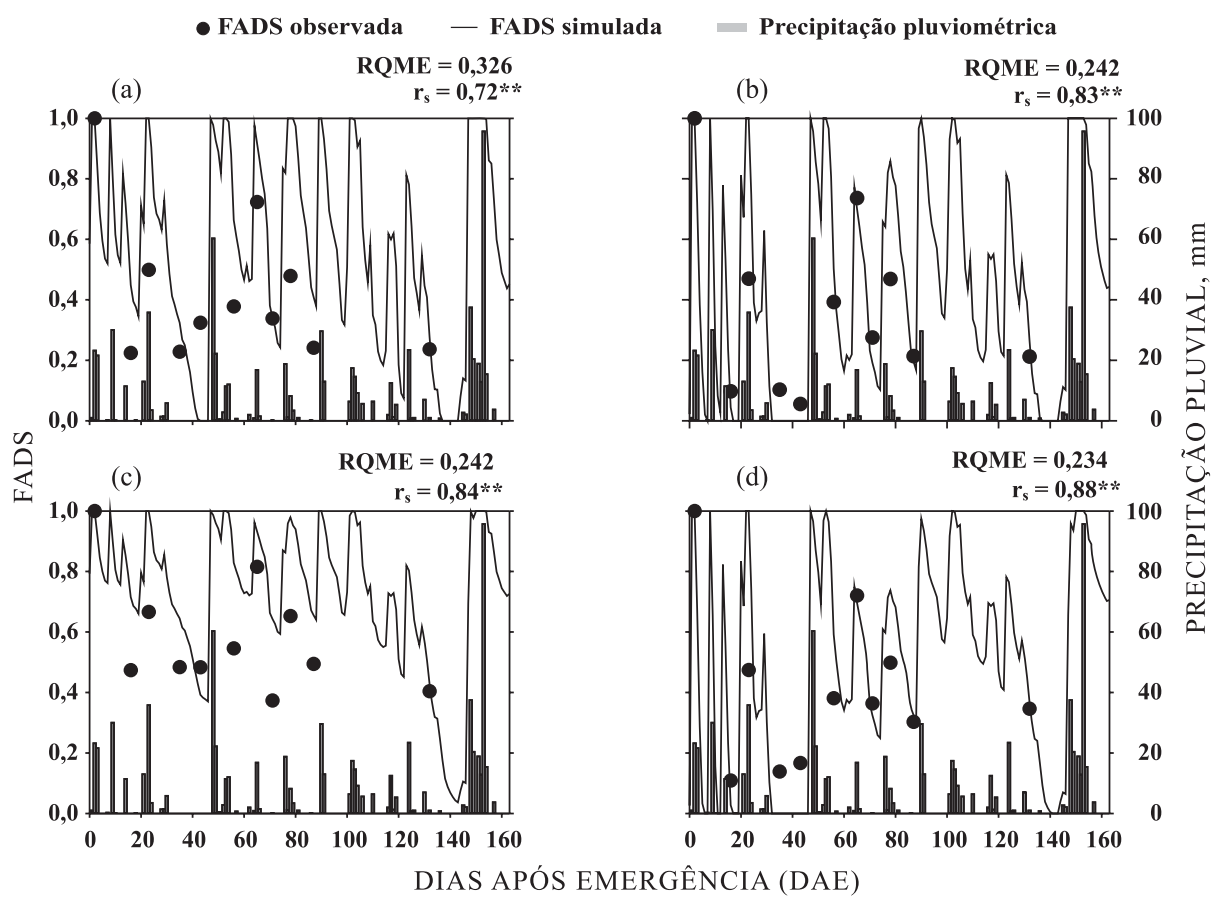

Figura 6. Fração de água disponível no solo (FADS) observada e simulada no perfil de solo onde ocorre crescimento radicular pelo modelo de Amir \& Sinclair (1991a,b) com RDmax de 0,30 m, modelo original (a) e modificado (b), e com RDmax de $0,60 \mathrm{~m}$, modelo original (c) e modificado (d), e precipitação pluvial (P, mm), para a semeadura de 18/04/2007 do cultivar BRS 177. Santa Maria, RS. RQME: raiz do quadrado médio do erro, $r_{\mathrm{s}}$ : teste de correlação não paramétrico de Spearman R. NS: não significativo, *significativo a $5 \%$. 
No modelo de Amir \& Sinclair (1991a,b), utilizando-se a profundidade do sistema radicular de 0,30 m e introduzindo-se o modelo de desenvolvimento e de crescimento radicular, resultou em melhor correlação e menor RQME para a data de semeadura de 7/07/2006, e a segunda maior correlação e o segundo menor valor RQME para a data de semeadura de 18/ 04/2007, indicando que as modificações foram apropriadas para as condições de clima e solo de Santa Maria.

Na semeadura do dia 7/07/2006, valores observados de FADS coletados nos dias 4/10/2006 (21 DAE), 10/10/2006 (27 DAE) e 19/10/2006 (36 DAE) foram próximos ao PMP, mesmo com as frequentes chuvas observadas no período (Figuras 2 e 5). Uma possível explicação para esses resultados é que a água precipitada teve baixa infiltração no solo. Este fato pode ser devido à compactação do solo na camada de 0,20-0,30 m ocasionada pelos vários anos de sistema convencional (aragens e gradagens) utilizados na área de estudo. Outra possibilidade é que a primeira chuva após a semeadura $(65,4 \mathrm{~mm}$ no dia 15/09/2006) provocou a formação de uma crosta superficial de solo (selamento superficial), que diminuiu a infiltração de água no solo, aumentou o escorrimento superficial e, consequentemente, diminuiu o conteúdo de água observado no solo.

Esta camada de solo compactada entre $0,20-0,30 \mathrm{~m}$ também pode explicar os valores de RQME mais baixos quando se usou RDmax de $0,30 \mathrm{~m}$ do que quando foi usado uma RDmax de 0,60 m em ambos os modelos (com exceção a Figura 6), pois diminui a penetração do sistema radicular no perfil do solo, mantendo-o restrito às primeiras camadas de solo, com poucas raízes atingindo as camadas mais profundas.

Ao se optar pelo modelo de Campbell \& Diaz (1988), o modelo modificado deve ser preferido em relação ao modelo original, apesar do modelo modificado não ter simulado melhor a FADS que o modelo original em algumas situações (Figura 1), pois tem a vantagem de não ser necessário o conhecimento prévio da data da maturidade fisiológica. Quando se utilizam diferentes datas de semeadura na cultura do trigo, a data da maturidade fisiológica pode variar de acordo com o cultivar utilizado, o que torna difícil o uso do modelo original de Campbell \& Diaz (1988). Na escolha de um modelo matemático, o critério de operacionalidade e do significado biológico dos coeficientes, bem como das funções de resposta, pode ser preferido ao critério da precisão da simulação, pois o primeiro dá ao usuário maior segurança para usar o modelo em regiões distintas daquelas em que o modelo foi desenvolvido.

O modelo modificado de Amir \& Sinclair (1991a,b) simulou melhor a FADS do que o modelo original em todas as situações, apresentando menores valores de RQME e maiores valores de correlação pelo teste não paramétrico de Spearman R. Assim, recomenda-se a utilização do modelo Amir \& Sinclair (1991a,b) modificado, pois esta versão alia melhora da predição com representação mais realista do ponto de vista biológico do crescimento radicular durante o ciclo de desenvolvimento da cultura.

Comparando-se as versões modificadas dos dois modelos, recomenda-se a utilização do modelo de Amir \& Sinclair (1991a,b) para a simulação do balanço de água no solo cultivado com trigo, por ser um modelo de fácil implantação e operacionalidade, realista do ponto de vista biológico, mais simples e com maior precisão do que o modelo de Campbell \& Diaz (1988). No entanto, o modelo de Campbell \& Diaz (1988) tem a particularidade de simular a interceptação pelo dossel de parte da água que precipita antes de chegar à superfície do solo, o que é uma maneira mecanística de simulação do fluxo de água da atmosfera até o interior do solo em agroecossistemas, e que não é levado em conta no modelo de Amir \& Sinclair (1991a,b). Assim, como a pesquisa inclui uma faixa grande de objetivos, desde a básica até a aplicada, pode-se usar o modelo de Campbell \& Diaz (1988) para entender melhor o sistema que está sendo modelado, na medida em que este modelo fornece informações sobre processos básicos intermediários do balanço de água no solo, como por exemplo, a percolação profunda e o escorrimento superficial.

\section{CONCLUSÕES}

1. A substituição da data de maturidade fisiológica do trigo pelo modelo de desenvolvimento Wang \& Engel - WE torna o modelo de balanço de água no solo de Campbell \& Diaz (1988) mais realista e com maior possibilidade de desempenho satisfatório em regiões de clima distinto daquele em que foi desenvolvido, tornando-o mais robusto e geral.

2. A substituição do modelo de soma térmica pelo modelo de desenvolvimento Wang \& Engel - WE e a introdução de um modelo de crescimento radicular tornam o modelo de Amir \& Sinclair (1991a,b) mais realista e melhoram a estimativa do balanço de água no solo.

3. Para a cultura do trigo, a utilização da profundidade máxima do sistema radicular de $0,30 \mathrm{~m}$ é mais apropriada para a simulação da fração de água disponível no solo, para a unidade de mapeamento de solo São Pedro.

4. Para Argissolo do Rio Grande do Sul, o modelo de Amir \& Sinclair foi melhor para estimar a fração de água disponível no solo para a cultura do trigo.

\section{AGRADECIMENTOS}

À CAPES, pela bolsa de doutorado ao autor Cleber Maus Alberto. Ao CNPq, pelas bolsas de iniciação científica PIBIC/CNPq/UFSM aos autores Felipe 
Brendler Oliveira e Alencar Junior Zanon, e de produtividade em pesquisa ao autor Nereu Augusto Streck. Os autores agradecem aos revisores ad hoc pelas valiosas contribuições.

\section{LITERATURA CITADA}

ALBERTO, C.M. Modelagem do desenvolvimento e do balanço de água no solo em trigo. Santa Maria, Universidade Federal de Santa Maria, 2008. 122p. (Tese de Doutorado)

AMIR, J. \& SINCLAIR, T.R. A model of the temperature and solar-radiation effects on spring wheat growth and yield. Field Crop Res., 29:47-58, 1991a.

AMIR, J. \& SINCLAIR, T.R. A model of water limitation on spring wheat growth and yield. Field Crop Res., 29:59-96, $1991 b$.

CAMPBELL, G.S. \& DIAZ, R. Simplified soil-water balance models to predict crop transpiration. In: BIDINGER, F.R. \& JOHANSEN, C. Drought research priorities for the dryland tropics. Parancheru, ICRISAT, 1988. p.15-26.

SOCIEDADE BRASILEIRA DE CIÊNCIA DO SOLO/ COMISSÃO DE QUÍMICA E FERTILIDADE DO SOLO SBCS/CQFS. Manual de adubação e calagem para os Estados do Rio Grande do Sul e Santa Catarina. 10.ed. Porto Alegre, 2004. 400p.

DARDANELLI, J.L.; RITCHIE, J.T.; CALMON, M.; ANDRIANI, J.M. \& COLLINO, D.J. An empirical model for root water uptake. Field Crop. Res., 87:59-71, 2004.

EITZINGER, J.; STASTNA, M.; ZALUD, Z. \& DUBROVSKY, M. A simulation study of the effect of soil water balance and water stress on winter wheat production under different climate change scenarios. Agric. Water Manag., 61:195-217, 2003.

EMPRESA BRASILEIRA DE PESQUISA AGROPECUÁRIA EMBRAPA. Manual de métodos de análise de solos. Rio de Janeiro, 1997. 212p.
GALVAGNI, G.A. Disponibilidade e crescimento de plantas de milho submetidas a déficit hídrico terminal. Santa Maria, Universidade Federal de Santa Maria, 1998. 81p. (Tese de Mestrado)

JANSSEN, P.H.M. \& HEUBERGER, P.S.C. Calibration of process-oriented models. Ecol. Model., 83:55-56, 1995.

MORENO, J.A. Clima do Rio Grande do Sul. Porto Alegre, Secretaria da Agricultura, 1961. 46p.

OKUYAMA, L.A.; FEDERIZZI, L.C. \& BARBOSA NETO, J.F. Correlation and path analysis of yield and its components and plant traits in wheat. Ci. Rural, 34:1701-1708, 2004.

SILVA, J.C.; HELDWEIN, A.B.; MARTINS, F.B. \& MAASS, G.F. Simulação para determinação das épocas de semeadura com menor risco de estresse hídrico para o feijão na região central do estado do Rio Grande do Sul. Irriga, 11:188-197, 2006.

STONE, L.F. \& SILVEIRA, P.M. Efeitos do sistema de preparo na compactação do solo, disponibilidade hídrica e comportamento do feijoeiro. Pesq. Agropec. Bras., 34:8391, 1999.

STRECK, N.A. Do we know how plants sense a drying soil? Ci. Rural, 2:581-584, 2004.

STRECK, N.A. \& ALBERTO, C.M. Simulação do impacto da mudança climática sobre a água disponível do solo em agroecossistemas de trigo, soja e milho em Santa Maria, RS. Ci. Rural, 36:424-433, 2006.

STRECK, N.A.; WEISS, A. \& BAENZIGER, P.S. A generalized vernalization response function for winter wheat. Agron. J., 95:155-159, 2003a.

STRECK, N.A.; WEISS, A.; XUE, Q. \& BAENZIGER, P.S. Improving predictions of developmental stages in winter wheat. Agric. For. Meteorol., 115:139-150, 2003b.

URACH, F.L. Estimativa da retenção de água em solos para fins de irrigação. Santa Maria, Universidade Federal de Santa Maria, 2007. 78p. (Tese de Mestrado)

WANG, E. \& ENGEL, T. Simulation of phenological development of wheat crops. Agric. Syst., 58:1-24, 1998.

ZADOKS, J.C.; CHANG, T.T. \& KONZAK, C.F. A decimal code for the growth stages of cereals. Weed Res., 14:415421, 1974. 